\title{
INTEGERS DIVISIBLE BY THE SUM OF THEIR PRIME FACTORS
}

\author{
JEAN-MARIE DE KONINCK AND FLORIAN LUCA
}

Abstract. For each integer $n \geqslant 2$, let $\beta(n)$ be the sum of the distinct prime divisors of $n$ and let $\mathcal{B}(x)$ stand for the set of composite integers $n \leqslant x$ such that $n$ is a multiple of $\beta(n)$. Upper and lower bounds are obtained for the cardinality of $\mathcal{B}(x)$.

§1. Introduction. Given an arithmetical function $f$ such that $f(n) \leqslant n$, it is somewhat natural to ask how often can $n$ be a multiple of $f(n)$. For instance, letting $\tau(n)$ (resp. $\omega(n)$ ) stand for the number of divisors (resp. number of distinct prime divisors) of $n$, Spiro [9] has shown that $\#\{n \leqslant x$ : $\tau(n) \mid n\}=(x / \sqrt{\log x})(\log \log x)^{-1+o(1)}$, while Cooper and Kennedy [2] showed that the set $\{n: \omega(n) \mid n\}$ has density 0 . More general results were obtained by Erdös and Pomerance [5], including the case $\Omega(n) \mid n$, where $\Omega(n)$ stands for the number of prime power divisors of $n$. Other results of this type have recently been obtained by Banks, Garaev, Luca and Shparlinski [1].

More recently, Vaughan and Weis [11] investigated the counting function of the set of composite integers $n$ such that $n-1$ is a multiple of $\sum_{p \mid n}(p-1)$. Here, we examine the counting function of the set of composite integers $n$ such that $n$ is a multiple of $\beta(n):=\sum_{p \mid n} p$.

§2. Main result. Let $\mathcal{B}(x):=\{n \leqslant x: n$ is composite and $\beta(n) \mid n\}$. We shall prove the following result.

THEOREM 1. For $x$ sufficiently large,

$$
x \exp \left\{-c_{1}(1+o(1)) \ell(x)\right\}<\# \mathcal{B}(x)<x \exp \left\{-c_{2}(1+o(1)) \ell(x)\right\},
$$

where $\ell(x):=\sqrt{\log x \log \log x}$ and $c_{1}$ and $c_{2}$ can be taken as $c_{1}=3 / \sqrt{2}$ and $c_{2}=1 / \sqrt{2}$.

§3. Preliminary results. Throughout this paper, we use the Vinogradov symbols 》 and « as well as the Landau symbols $O$ and $o$ with their regular meanings. For each integer $n \geqslant 2$, let $P(n)$ stand for the largest prime factor of $n$, and set $P(1)=1$.

LEMMA 1. For every $\varepsilon>0$, there exists a real number $x_{\varepsilon}$ such that, if $x>x_{\varepsilon}$, then the interval $[x / 2, x]$ contains at least $x^{1-\varepsilon}$ distinct integers of the form $m-\beta(m)$, for some positive integer $m \leqslant x$ such that $\omega(m)<2 \log \log x$. 
Proof. Let $\varepsilon$ be fixed in the interval $(0,1)$. For large $x$, the interval $[2 x / 3, x]$ contains $(1+o(1)) \rho(2 / \varepsilon) x / 3$ positive integers $m$ such that $P(m)<x^{\varepsilon / 2}$. Here, for a positive real number $u>1, \rho(u)$ stands for the Dickman function (see, for example, Theorem 6 on page 367 in [10]). Since the number of positive integers $m \leqslant x$ for which $\omega(m) \geq 2 \log \log x$ is $o(x)$ (because the function $\omega(m)$ has a normal order equal to $\log \log x$ for $m$ in the interval $[1, x]$ ), it follows that most of the above numbers $m$ have $\omega(m)<2 \log \log x$. Note that, if $m$ is such an integer, then $\beta(m) \ll x^{\varepsilon / 2} \log \log x$. Therefore, since $m>2 x / 3$, it follows that $m-\beta(m)>x / 2$ if $x$ is large enough. Thus, for such numbers $m$, we have $m-\beta(m) \in[x / 2, x]$. Let $m$ be one such number and assume that $m-\beta(m)=m^{\prime}-\beta\left(m^{\prime}\right)$ for some $m^{\prime} \neq m$. Then

$$
\left|m-m^{\prime}\right|=\left|\beta(m)-\beta\left(m^{\prime}\right)\right| \ll x^{\varepsilon / 2} \log \log x .
$$

This argument shows that, for a fixed $m$, there are no more than $O\left(x^{\varepsilon / 2} \log \log x\right)$ values of $m^{\prime}$ for which $m-\beta(m)=m^{\prime}-\beta\left(m^{\prime}\right)$ might hold.

In particular, the number of distinct values of the form $m-\beta(m)$ for such $m$ is

$$
\gg \frac{x}{x^{\varepsilon / 2} \log \log x}=\frac{x^{1-\varepsilon / 2}}{\log \log x}>x^{1-\varepsilon} \quad \text { when } x>x_{\varepsilon},
$$

which implies the conclusion of Lemma 1.

§4. Proof of Theorem 1. Let $x$ be a large number.

Let $y=y(x)$ be a function tending to $+\infty$ with $x$ that we shall determine later. We put $u=\log x / \log y$. Recall that a positive integer $m$ is powerful if $p^{2} \mid m$ whenever $p$ is a prime factor of $m$.

Let

$$
\begin{aligned}
& \mathcal{B}_{1}(x)=\{n \in \mathcal{B}(x): P(n) \leqslant y\} \\
& \mathcal{B}_{2}(x)=\left\{n \in \mathcal{B}(x) \backslash \mathcal{B}_{1}(x): \omega(n) \geqslant u\right\} ; \\
& \mathcal{B}_{3}(x)=\left\{n \in \mathcal{B}(x): m \mid n \text { holds for some powerful } m>y^{2}\right\} ; \\
& \mathcal{B}_{4}(x)=\mathcal{B}(x) \backslash\left(\mathcal{B}_{1}(x) \cup \mathcal{B}_{2}(x) \cup \mathcal{B}_{3}(x)\right) .
\end{aligned}
$$

We shall be using the well-known estimate

$$
\Psi(x, y):=\#\{n \leqslant x: P(n) \leqslant y\}=x \exp \{-(1+o(1)) u \log u\},
$$

which holds in the range $(\log \log x)^{5 / 3+\varepsilon} \leqslant \log y \leqslant \log x$ for any fixed small $\varepsilon>0$, a result due to Hildebrand [6].

In view of (2), we have

$$
\# \mathcal{B}_{1}(x) \leqslant x \exp \{-(1+o(1)) u \log u\} .
$$

We shall assume from now on that $n \notin \mathcal{B}_{1}(x)$.

Let $z$ be any positive real number.

By writing each integer $n$ with $\omega(n) \geqslant z$ as $n=p_{1} p_{2} \cdots p_{\lfloor z\rfloor} m$ for some positive integer $m$ and some distinct primes $p_{1}, p_{2}, \cdots, p_{\lfloor z\rfloor}$, we have that $m$ can take at most $\left\lfloor x / p_{1} p_{2} \cdots p_{\lfloor z\rfloor}\right\rfloor$ values. Hence, using Stirling's formula, as 
well as the fact that

$$
\sum_{p \leqslant y} \frac{1}{p}=\log \log y+O(1)
$$

holds as $y$ tends to infinity, we get that

$$
\begin{aligned}
\#\{n \leqslant x: \omega(n) \geqslant z\} & \leqslant \sum_{p_{1} \ldots p_{\lfloor z\rfloor} \leqslant x} \frac{x}{p_{1} \ldots p_{\lfloor z\rfloor}} \leqslant \frac{x}{\lfloor z\rfloor !}\left(\sum_{p \leqslant x} \frac{1}{p}\right)^{\lfloor z\rfloor} \\
& \leqslant x\left(\frac{e \log \log x+O(1)}{\lfloor z\rfloor}\right)^{\lfloor z\rfloor} \\
& \leqslant x \exp \{-(1+o(1)) z \log z\}
\end{aligned}
$$

provided that $z$ is much larger than $\log \log x$, for instance when $\log \log \log x=$ $o(\log z)$. Hence, choosing $z=u$, it follows from (4) that

$$
\# \mathcal{B}_{2}(x)=\#\{n \leqslant x: \omega(n) \geqslant u\} \leqslant x \exp \{-(1+o(1)) u \log u\},
$$

provided that $\log \log \log x=o(\log u)$. From here on, we assume that $n \notin \mathcal{B}_{1}(x) \cup \mathcal{B}_{2}(x)$.

Clearly,

$$
\# \mathcal{B}_{3}(x) \leqslant \sum_{\substack{m>y^{2} \\ m \text { powerful }}} \frac{x}{m} \leqslant \frac{x}{y},
$$

where the above inequality follows by partial summation from the known fact that the estimate

$$
\#\{m \leqslant x: m \text { powerful }\}=C_{1} \sqrt{x}+O\left(x^{1 / 3}\right)
$$

holds as $x$ tends to infinity (see, for example, Theorem 14.4 in [7]).

It remains to estimate $\# \mathcal{B}_{4}(x)$. We first make some comments about the integers in $\mathcal{B}_{4}(x)$. Write $n=n_{1} n_{2}$, where $\operatorname{gcd}\left(n_{1} n_{2}\right)=1, n_{1}$ is powerful and $n_{2}$ is square-free. Since $n_{1} \leqslant y^{2}$ (because $n$ is not in $\mathcal{B}_{3}(x)$ ) and $P(n)>y$ (because $n$ is not in $\left.\mathcal{B}_{1}(x)\right)$, we get that $P(n) \mid n_{2}$. In particular, $P(n) \| n$. Secondly,

$$
\tau(n)=\tau\left(n_{1}\right) \tau\left(n_{2}\right) .
$$

Clearly, $\tau\left(n_{2}\right) \leqslant 2^{\omega(n)} \leqslant 2^{u}=\exp (O(u))$, because $n$ is not in $\mathcal{B}_{2}(x)$. Finally, it is well known that

$$
\tau\left(n_{1}\right)=\exp \left(O\left(\frac{\log n_{1}}{\log \log n_{1}}\right)\right)=\exp \left(O\left(\frac{\log y}{\log \log y}\right)\right) .
$$

In particular,

$$
\tau(n) \leqslant \exp (O(u)+o(\log y)) .
$$

We now let $n \in \mathcal{B}_{4}(x)$ and write $n=P(n) m$, where $m \leqslant x / y$ is a positive integer. Note that $m>1$, because $n$ is a composite. Let $d \mid n$ be such that $d=\beta(n)$. Reducing this equation modulo $P(n)$, we get that

$$
\begin{cases}\sum_{p \mid m} p \equiv 0 & (\bmod P(n)), \text { if } P(n) \mid d, \\ \sum_{p \mid m} p-d \equiv 0 & (\bmod P(n)), \text { otherwise, that is, if } d \mid m\end{cases}
$$


In the first case, $P(n)$ can take at most $\omega(\beta(m))=O(\log \beta(m))=O(\log x)$ values, once $m$ is fixed. (Note that $\beta(m)>0$ because $n$ is not a prime power.) In the second case, $P(n)$ can take again at most $O(\log x)$ values once $m$ and $d(d \mid m)$ are fixed. In conclusion, for a fixed value of $m$, the total number of values of $P(n)$ is

$$
\ll \tau(m) \log x \leqslant \tau(n) \log x=\exp (O(u)+o(\log y)),
$$

where we used the fact that $\log \log x=o(u)$, because $\log \log \log x=o(\log u)$. Since $m \leqslant x / y$, it follows that

$$
\# \mathcal{B}_{4}(x) \ll \frac{x}{y} \exp (O(u)+o(\log y)) .
$$

In order to optimize the bounds obtained in (3), (5), (6) and (8), we choose $u$ in such a way that $\log y=u \log u$. We then get

$$
\log y=\frac{\log x}{\log y} \log \left(\frac{\log x}{\log y}\right),
$$

so that

$$
\log ^{2} y=\frac{1}{2}(1+o(1)) \log x \log \log x
$$

giving

$$
\log y=(1+o(1)) \sqrt{\frac{1}{2} \log x \log \log x},
$$

and therefore

$$
u \log u=(1+o(1)) \sqrt{\frac{1}{2} \log x \log \log x} .
$$

The upper bound claimed by Theorem 1 follows now immediately from (3), (5), (6) and (8).

We now turn to the lower bound.

Let again $x$ be a large number. Then let $y=y(x)<x$ be some function of $x$ which tends to $+\infty$ with $x$ and will be determined later.

Let $\varepsilon>0$ be arbitrary in the interval $(0,1)$ but fixed. By Lemma 1 , the interval $[y / 2, y]$ contains at least $y^{1-\varepsilon}$ positive integers of the form $m-\beta(m)$, for some $m \leqslant y$ such that $\omega(m) \leqslant 2 \log \log y$. Let $M=m-\beta(m)$ be one of these integers. Let $k$ be a large positive integer having the same parity as $M$ and let

$$
I_{k}=\left[\frac{M}{3 k}, \frac{2 M}{3 k}\right]
$$

and

$$
J=\left[\frac{M}{3}, \frac{2 M}{3}\right] .
$$

Let $\left(m_{1}, m_{2}, \ldots, m_{k}\right)$ be a $k$-tuple of odd integers such that $m_{i} \in I_{k}$ for $1 \leqslant i \leqslant$ $k-1$ and $m_{k} \in J$, and such that $M=m_{1}+m_{2}+\cdots+m_{k}$. 
It is clear that the number of such representations is at least $\left(c_{4} M / k\right)^{k-1}$, where $c_{4}$ can be taken to be a constant smaller than $1 / 12$, once $y$ (and hence $x$ ) is large enough. Hence, let us choose $c_{4}=1 / 13$.

Assuming that $k=o(y)$, and using Vinogradov's Three Primes Theorem (see, for example, [8]), each one of the numbers $m_{i}$ can be written as a sum of three odd primes, and the number of such representations $m_{i}=p_{i, 1}+p_{i, 2}+p_{i, 3}$ is

$$
\gg \frac{m_{i}^{2}}{\log ^{3} m_{i}} \gg\left(\frac{y}{k}\right)^{2} \frac{1}{(\log (y / k))^{3}} .
$$

This argument shows that the number $L$ of $3 k$-tuples of primes

$$
\left(p_{i, 1}, p_{i, 2}, p_{i, 3}\right)_{1 \leqslant i \leqslant k}
$$

such that

$$
M=\sum_{i=1}^{k} \sum_{j=1}^{3} p_{i, j}
$$

satisfies

$$
L \gg\left(\frac{c_{5} y}{k}\right)^{3 k-1} \frac{1}{(\log (y / k))^{3 k}},
$$

with some positive constant $c_{5}$.

We now discard those $3 k$-tuples of primes such that either there exists one component $p_{i, j} \mid m$, or there exist $\left(i_{1}, j_{1}\right) \neq\left(i_{2}, j_{2}\right)$ such that $p_{i_{1}, j_{1}}=p_{i_{2}, j_{2}}$. We count the number of excluded $3 k$-tuples.

We handle the first case. Let $\left(i_{0}, j_{0}\right)$ be a fixed position and let $p=p_{i_{0}, j_{0}}$ be a fixed prime factor of $m$. The first $3 k-2$ components $(i, j)$ with $(i, j) \neq\left(i_{0}, j_{0}\right)$ can each be chosen in at most $\pi\left(I_{k}\right)$ ways, where $\pi\left(I_{k}\right)$ denotes the number of primes in $I_{k}$, and once those components have been chosen, the last one is uniquely determined.

Since $\left(i_{0}, j_{0}\right)$ can be chosen in $3 k$ ways and $p$ can be chosen in $\omega(m) \leqslant$ $2 \log \log y$ ways, it follows that the total number $U$ of such possibilities satisfies

$$
U \leqslant 6 k(\log \log y)\left(\pi\left(I_{k}\right)\right)^{3 k-2} \ll k \log \log y\left(\frac{c_{6} y}{k}\right)^{3 k-2} \frac{1}{(\log (y / k))^{3 k-2}} .
$$

Here, we may take $c_{6}$ to be any constant greater than $1 / 3$ once $y$ (and hence $x$ ) is large enough. Comparing equation (9) and (10), we observe that

$$
U \ll L\left(\frac{c_{6}}{c_{5}}\right)^{3 k-2} \frac{k^{2}(\log \log y)(\log (y / k))^{2}}{y}=o(U),
$$

where the last estimate above holds provided that $k=o(\log y)$.

Hence, assuming that this last condition is fulfilled, it follows that most of our $3 k$-tuples of primes constructed in this manner have the property that none of its components is a divisor of $m$.

We next count those $3 k$-tuples such that $p_{i_{1}, j_{1}}=p_{i_{2}, j_{2}}=p$. We see that the prime $p$ can be chosen in at most $\pi\left(I_{k}\right)$ ways, and the first $3 k-3$ primes $p_{i, j}$ for the locations $(i, j) \neq\left(i_{1}, j_{1}\right)$ and $(i, j) \neq\left(i_{2}, j_{2}\right)$ can also be chosen in at most $\pi\left(I_{k}\right)$ ways each, and once all such components have been chosen, the last one 
is uniquely determined. Since the pairs $\left(i_{1}, j_{1}\right)$ and $\left(i_{2}, j_{2}\right)$ can be chosen in at most $O\left(k^{2}\right)$ ways, it follows that the total number $V$ of such $3 k$-tuples satisfies the inequality

$$
V \ll k^{2} \pi\left(I_{k}\right)^{3 k-2} \ll k^{2}\left(\frac{c_{6} y}{k}\right)^{3 k-2} \frac{1}{(\log (y / k))^{3 k-2}} .
$$

Comparing equations (11) and (9), we see that

$$
V \ll L\left(\frac{c_{6}}{c_{5}}\right)^{3 k-2} \frac{k^{3}(\log (y / k))^{2}}{y}=o(L),
$$

where the last estimate above holds again because $k=o(\log y)$.

Hence, we conclude that a positive proportion (in fact, most of them) of our $3 k$-tuples of primes have mutually distinct components which do not divide $m$. We now consider numbers $n$ of the form $n=m \prod_{i=1}^{k} \Pi_{j=1}^{3} p_{i, j}$.

By the above argument and unique factorization, for fixed $m$ the number of such integers $n$ is, using (9),

$$
\gg \frac{1}{(3 k) !} L \gg \frac{1}{(3 k) !}\left(\frac{c_{5} y}{k}\right)^{3 k-1} \frac{1}{(\log (y / k))^{3 k}} .
$$

We now use Lemma 1 and vary $m$ in such a way that the integers $M=m-\beta(m)$ are all distinct, to get a total $W$ of pairs $(n, m)$, with

$$
W \gg y^{1-\varepsilon} \frac{1}{(3 k) !} L \gg \frac{1}{(3 k) !}\left(\frac{c_{5} y}{k}\right)^{3 k-\varepsilon} \frac{1}{(\log (y / k))^{3 k}} .
$$

Using Stirling's formula, we obtain

$$
W \gg \frac{c_{7}^{3 k} y^{3 k-\varepsilon}}{k^{6 k+\frac{1}{2}}(\log (y / k))^{3 k}},
$$

where we can take $c_{7}=c_{5} e / 3$. It is clear that these $n$ belong to $\mathcal{B}(x)$, because

$$
\beta(n)=\beta(m)+\sum_{i=1}^{k} \sum_{j=1}^{3} p_{i, j}=m,
$$

and $m \mid n$.

Unfortunately, not all integers $n$ which we counted in this way are distinct, because the same integer $n$ may appear from two distinct values of $m$. To bound the number of over-counts, we let $t=\omega(n)$. We know that $t=3 k+\ell$, where $\ell=\omega(m) \leqslant 2 \log \log y$.

We note that $m$ is determined by choosing a subset of $3 k$ prime factors of $n$. Hence, the maximal number $T$ of over-counts of the same number $n$ satisfies

$$
T \leqslant\left(\begin{array}{c}
t \\
3 k
\end{array}\right)=\left(\begin{array}{c}
3 k+\ell \\
3 k
\end{array}\right)=\left(\begin{array}{c}
3 k+\ell \\
\ell
\end{array}\right)=\exp \{O((\log k)(\log \log y))\},
$$

where the last inequality holds provided that $\ell \leqslant 2 \log \log y=o(k)$.

Thus, using (12), the number of distinct $n$ is at least

$$
\frac{W}{T} \gg \frac{c_{7}^{3 k} y^{3 k-\varepsilon}}{k^{6 k+\frac{1}{2}}(\log (y / k))^{3 k} \exp \{O((\log k)(\log \log y))\}} .
$$


The largest such integer $n$ does not exceed

$$
y\left(\frac{y}{k}\right)^{3 k-3} y^{3}=\frac{y^{3 k+1}}{k^{3 k-3}}:=x .
$$

Thus, by (13),

$$
\begin{aligned}
\# \mathcal{B}(x) \gg & \frac{y^{3 k+1}}{k^{3 k-3}} \frac{c_{7}^{k}}{k^{3 k+7 / 2} y^{1+\varepsilon}(\log (y / k))^{3 k} \exp \{O((\log k)(\log \log y))\}}, \\
= & x \exp \{-3 k \log k-(1+\varepsilon) \log y-3 k \log \log (y / k) \\
& +O(k+\log k \log \log y)\} .
\end{aligned}
$$

The above formula suggests choosing $k$ in terms of $y$ in such a way that the main term inside the above exponential is as small as possible.

Thus, we choose $k$ such that $k=\left\lfloor k^{\prime}\right\rfloor$ with

$$
3 k^{\prime} \log k^{\prime}=(1+\varepsilon) \log y .
$$

With this choice, we have

$$
k=\left(\frac{1+\varepsilon}{3}\right) \frac{\log y}{\log \log y}(1+o(1)),
$$

and

$$
\begin{aligned}
& 3 k \log k+(1+\varepsilon) \log y+3 k \log \log (y / k)+O(k+\log k \log \log y) \\
& \quad=3(1+\varepsilon) \log y+O\left(\frac{\log y}{\log \log y}\right) .
\end{aligned}
$$

To express $y$ in terms of $x$, we take the logarithm of both sides of equation (14), thus obtaining

$$
(3 k+1) \log y-(3 k-3) \log k=\log x,
$$

which together with (16) leads to

$$
(1+\varepsilon) \frac{\log ^{2} y}{\log \log y}(1+o(1)) \log x
$$

which gives

$$
\log y=\sqrt{\frac{1}{2(1+\varepsilon)}}(1+o(1)) \sqrt{\log x \log \log x} .
$$

Combining (15), (16) and (17), we thus get that

$$
\# \mathcal{B}(x) \geqslant x \exp \left\{-(1+o(1)) \frac{3}{\sqrt{2}} \sqrt{(1+\varepsilon) \log x \log \log x}\right\},
$$

and letting $\varepsilon \rightarrow 0$, we get the desired lower bound.

Remark 1. Arguing heuristically, one could say that the probability that $\beta(n) \mid n$ for some integer $n$ which is not a prime power should be approximately $1 / \beta(n)$, in which case $\# \mathcal{B}(x)$ should be close to $\sum_{\substack{2 \leqslant n \leqslant x \\ n \text { composite }}} \frac{1}{\beta(n)}$. But this last sum 
was investigated by Xuan [12], who obtained that

$$
\sum_{2 \leqslant n \leqslant x} \frac{1}{\beta(n)}=(D+o(1)) \sum_{2 \leqslant n \leqslant x} \frac{1}{P(n)}
$$

for some positive constant $D<1$. On the other hand, it was shown by Erdös, Ivić and Pomerance [4] that

$$
\sum_{2 \leqslant n \leqslant x} \frac{1}{P(n)}=x \exp \{-(1+o(1)) \sqrt{2 \log x \log \log x}\} .
$$

Hence, in view of these estimates and since

$$
\sum_{\substack{2 \leqslant n \leqslant x \\ n \text { prime power }}} \frac{1}{\beta(n)} \leqslant \sum_{p \leqslant x}\left\lfloor\frac{\log x}{\log p}\right\rfloor \frac{1}{p}=O(\log x),
$$

one may conclude that

$$
\sum_{\substack{2 \leqslant n \leqslant x \\ n \text { composite }}} \frac{1}{\beta(n)}=x \exp \{-(1+o(1)) \sqrt{2 \log x \log \log x}\} .
$$

Comparing this last estimate with the bounds obtained in Theorem 1, it is somewhat reassuring to observe that indeed we have $c_{1}=1 / \sqrt{2}<\sqrt{2}<c_{2}=$ $3 / \sqrt{2}$.

Remark 2. Theorem 1 suggests that there might exist a constant $c$ such that the estimate

$$
\sum_{\substack{n \leq x \\ \beta(n) \mid n}} 1=x \exp \{-c(1+o(1)) \sqrt{\log x \log \log x}\}
$$

holds as $x \rightarrow \infty$. We could not succeed in proving such an estimate, but, in light of Remark 1, we conjecture that $c=\sqrt{2}$.

Remark 3. There are a few papers in the literature in which the average prime divisor of a positive integer $n$ has been investigated (see [1] and [3], for example). This is defined as $P^{*}(n)=\beta(n) / \omega(n)$. The method of proof of Theorem 1 can easily be extended to give upper and lower bounds on the cardinality of the set $\mathcal{N}(x)=\left\{n \leqslant x: n\right.$ composite and $\left.P^{*}(n) \mid n\right\}$. Note that $P^{*}(n)$ is only a rational number, and by the divisibility relation $P^{*}(n) \mid n$ we mean that $n / P^{*}(n)=n \omega(n) / \beta(n)$ is an integer. Clearly, if $n / \beta(n)$ is an integer, then $n \omega(n) / \beta(n)$ is an integer as well. Hence, $\# \mathcal{N}(x)$ is at least as large as the left side of inequality (1). The fact that $\# \mathcal{N}(x)$ is at most as large as the right side of inequality (1) follows from an argument similar to the one used in the proof of Theorem 1. We give no further details.

Acknowledgements. The first author was partially supported by a grant from NSERC, while the second author was supported by grant PAPIITIN104505 from the UNAM. Both authors were supported by grants from the Groupe de travail Québec-Mexique 2004-2006 avec l'appui du Ministére des relations internationales and the Secretaria de Relationes Exteriores, Mexico. The authors would also like to thank Gang Yu for some useful comments. 


\section{References}

1. W. Banks, M. Z. Garaev, F. Luca and I. E. Shparlinski, Uniform distribution of the fractional part of the average prime divisor, Forum Math. 17 (2005), 885-901.

2. C. N. Cooper and R. E. Kennedy, Chebychev's inequality and natural density, Amer. Math. Monthly 96 (1989), 118-124.

3. J. M. De Koninck and A. Ivić, The distribution of the average prime divisor of an integer, Arch. Math. (Basel), 43 (1984), 37-43.

4. P. Erdős, A. Ivić and C. Pomerance, On sums involving reciprocals of the largest prime factor of an integer, Glasgow Math. Ser. III 21 (41) (1986), 283-300.

5. P. Erdős, and C. Pomerance, On a theorem of Besikovitch: values of arithmatic functions that divide their argument, Indian J. Math. 32 (1990), 279-287.

6. A. Hildebrand, On the number of positive integers $\leqslant x$ and free of prime factors $>y$, J. Number Theory 22 (1986), 289-307.

7. A. Ivić, The Riemann-Zeta Function, Theory and Applications, Dover Publications (Mineola, New York, 2003).

8. K. Ramachandra and A. Sankaranarayanan, Vinogradov's Three Primes Theorem, Math. Student 66 (1997), 1-4 and 27-72.

9. C. Spiro, How often is the number of divisors of $n$ a divisor of $n$ ?, J. Number Theory 21 (1985), $81-100$.

10. G. Tenenbaum, Introduction to Analytic and Probabilistic Number Theory, Cambridge University Press (1995).

11. R. C. Vaughan and K. L. Weis, On sigma-phi numbers, Mathematika 48 (2001), 169-189.

12. T. Z. Xuan, On sums involving reciprocals of certain large additive functions, Publ. Inst. Math. (Beograd) (N.S.) 45 (59) (1989), 41-55.

Jean-Marie De Koninck,

Département de mathématiques,

Université Laval,

Québec G1K 7P4,

Canada.

E-mail: jmdk@mat.ulaval.ca

Florian Luca,

Mathematical Institute, UNAM,

Ap. Postal 61-3 (Xangari), CP 58 089,

Morelia, Michoacán,

Mexico.

E-mail: fluca@matmor.unam.mx

\section{MSC (2000): Primary, 11A41;}

Secondary, 11A25.

Received on the 20th of June, 2005 